\title{
On the coordinate representation of NLO BFKL *
}

\author{
V.S. Fadin ${ }^{a \dagger}$, R. Fiore ${ }^{b \ddagger}$, A. Papa ${ }^{b \dagger}$ \\ ${ }^{a}$ Budker Institute of Nuclear Physics, 630090 Novosibirsk, Russia \\ Novosibirsk State University, 630090 Novosibirsk, Russia \\ ${ }^{b}$ Dipartimento di Fisica, Università della Calabria, \\ Istituto Nazionale di Fisica Nucleare, Gruppo collegato di Cosenza, \\ Arcavacata di Rende, I-87036 Cosenza, Italy
}

\begin{abstract}
The "non-Abelian" part of the quark contribution to the BFKL kernel in the nextto-leading order (NLO) is found in the coordinate representation by direct transfer of the contribution from the momentum representation where it was calculated before. The results obtained are used for the examination of conformal properties of the NLO BFKL kernel and of the relation between the BFKL and color dipole approaches.
\end{abstract}

${ }^{*}$ Work supported in part by the Russian Fund of Basic Researches and in part by Ministero Italiano dell'Istruzione, dell'Università e della Ricerca.

$\dagger_{e}$-mail address: FADIN@INP.NSK.SU

${ }^{\ddagger}$-mail address: FIORE@CS.INFN.IT

${ }^{\dagger} e$-mail address: PAPA@CS.INFN.IT 


\section{Introduction}

The BFKL approach [1, based on the gluon Reggeization, gives a common basis for the theoretical description of high energy processes with fixed (not growing with energy) momentum transfers. This approach is well developed now in the next-to-leading approximation (NLA). In particular, the kernel of the BFKL equation is found in the next-to-leading order (NLO) not only for the forward scattering [2], i.e. for $t=0$ and the color singlet in the $t$-channel, but also for arbitrary momentum transfer $t$ and any possible color state in the $t$-channel [3, 4, 5]. Particularly interesting is the color singlet state, which is considered below, because physical particles are colorless. All the results mentioned above are obtained in the momentum representation. There are at least two reasons for considering the singlet BFKL kernel in the coordinate representation in the transverse space.

First, just in this representation the BFKL equation in the leading approximation exhibits the famous property of conformal invariance [6], which is extremely important for finding solutions of the equation. Therefore conformal properties of the NLO BFKL are very interesting. Evidently, the conformal invariance is violated in the NLA by the renormalization; the question is if the renormalization is the only source of violation.

Second, it is the representation in which the color dipole approach to high energy scattering [7, very popular now, is formulated. An advantage of the color dipole approach is a clear physical picture of the high energy processes. Moreover, this approach is naturally applied not only at low parton densities, but also in the saturation regime [8], where equations of evolution of parton densities with energy become nonlinear. In general, there is an infinite hierarchy of coupled equations [9, 10. In the simplest case, when the target is a large nucleus, it is reduced to the BK (Balitsky-Kovchegov) equation [9]. A clear understanding of the relation between these two approaches is very important. It could help in further development of the theoretical description of small- $x$ processes. Unfortunately, the "native" representations for these two approaches are different: for the color dipole approach it is the coordinate representation in the transverse space, whereas for the BFKL approach it is the momentum one. Therefore the relation between these two approaches is not quite transparent, although it is affirmed [7, 9], that in the linear regime the color dipole gives the same results as the BFKL approach for the color singlet channel.

The leading order color singlet BFKL kernel has been investigated in the coordinate representation in details [6] before the advent of the dipole approach. The relation between BFKL and color dipole in the leading order was analyzed recently in Ref. [11]. In this paper we extend this analysis to the NLO. We plan to obtain both quark and gluon parts of the kernel in the dipole approach by direct transformation of the BFKL kernel in the momentum representation to the coordinate representation. In this paper we consider the "non-Abelian" part of the quark contribution to the NLO BFKL kernel. 


\section{The leading order kernel in coordinate representation}

In this Section we consider the BFKL kernel at the leading order. We will use the following notation: $\vec{q}_{i}^{\prime}$ and $\vec{q}_{i}, i=1,2$, represent the transverse momenta of Reggeons in initial and final $t$-channel states, while $\vec{r}_{i}^{\prime}$ and $\vec{r}_{i}$ are the corresponding conjugate coordinates. The state normalization is

$$
\left\langle\vec{q} \mid \vec{q}^{\prime}\right\rangle=\delta\left(\vec{q}-\vec{q}^{\prime}\right), \quad\left\langle\vec{r} \mid \vec{r}^{\prime}\right\rangle=\delta\left(\vec{r}-\vec{r}^{\prime}\right),
$$

so that

$$
\langle\vec{r} \mid \vec{q}\rangle=\frac{e^{i \vec{q} \vec{r}}}{(2 \pi)^{1+\epsilon}},
$$

where $\epsilon=(D-4) / 2 ; D-2$ is the dimension of the transverse space and is taken different from 2 for the regularization of divergences. Note that in our previous papers we denoted the initial (final) momenta as $\vec{q}_{1}$ and $-\vec{q}_{1}^{\prime}\left(\vec{q}_{2}\right.$ and $\left.-\vec{q}_{2}^{\prime}\right)$ and used the normalization $\left\langle\vec{q} \mid \vec{q}^{\prime}\right\rangle=\vec{q}^{2} \delta\left(\vec{q}-\vec{q}^{\prime}\right)$. We will use also the notation $\vec{q}=\vec{q}_{1}+\vec{q}_{2}, \quad \vec{q}^{\prime}=\vec{q}_{1}^{\prime}+\vec{q}_{2}^{\prime} ; \quad \vec{k}=\vec{q}_{1}-\vec{q}_{1}^{\prime}=\vec{q}_{2}^{\prime}-\vec{q}_{2}$. The BFKL kernel in the operator form is written as

$$
\hat{\mathcal{K}}=\hat{\omega}_{1}+\hat{\omega}_{2}+\hat{\mathcal{K}}_{r},
$$

where

$$
\left\langle\vec{q}_{i}\left|\hat{\omega}_{i}\right| \vec{q}_{i}^{\prime}\right\rangle=\delta\left(\vec{q}_{i}-\vec{q}_{i}^{\prime}\right) \vec{q}_{i}^{2} \omega\left(-\vec{q}_{i}^{2}\right),
$$

with $\omega(t)$ the gluon Regge trajectory, and $\hat{\mathcal{K}}_{r}$ represents real particle production in Reggeon collisions. The $s$-channel discontinuities of scattering amplitudes for the processes $A+B \rightarrow$ $A^{\prime}+B^{\prime}$ have the form

$$
-4 i(2 \pi)^{D-2} \delta\left(\vec{q}_{A}-\vec{q}_{B}\right) \operatorname{disc}_{s} \mathcal{A}_{A B}^{A^{\prime} B^{\prime}}=\left\langle A^{\prime} \bar{A}\left|e^{Y \hat{\mathcal{K}}} \frac{1}{\hat{\vec{q}}_{1}^{2} \hat{\vec{q}}_{2}^{2}}\right| \bar{B}^{\prime} B\right\rangle .
$$

In this equation $Y=\ln \left(s / s_{0}\right), s_{0}$ is an appropriate energy scale, $\quad q_{A}=p_{A^{\prime}}-p_{A}, \quad q_{B}=p_{B}-p_{B^{\prime}}$, and

$$
\begin{aligned}
& \left\langle\vec{q}_{1}, \vec{q}_{2}|\hat{\mathcal{K}}| \vec{q}_{1}^{\prime}, \vec{q}_{2}^{\prime}\right\rangle=\delta\left(\vec{q}-\vec{q}^{\prime}\right) \frac{1}{\vec{q}_{1}^{2} \vec{q}_{2}^{2}} \mathcal{K}\left(\vec{q}_{1}, \vec{q}_{1}^{\prime} ; \vec{q}\right), \\
& \left\langle\vec{q}_{1}, \vec{q}_{2} \mid \vec{B}^{\prime} B\right\rangle=4 p_{B}^{-} \delta\left(\vec{q}_{B}-\vec{q}_{1}-\vec{q}_{2}\right) \Phi_{B^{\prime} B}\left(\vec{q}_{1}, \vec{q}_{2}\right), \\
& \left\langle A^{\prime} \bar{A} \mid \vec{q}_{1}, \vec{q}_{2}\right\rangle=4 p_{A}^{+} \delta\left(\vec{q}_{A}-\vec{q}_{1}-\vec{q}_{2}\right) \Phi_{A^{\prime} A}\left(\vec{q}_{1}, q_{2}\right) .
\end{aligned}
$$

where $p^{ \pm}=\left(p_{0} \pm p_{z}\right) / \sqrt{2}$; the kernel $\mathcal{K}\left(\vec{q}_{1}, \vec{q}_{1}^{\prime} ; \vec{q}\right)$ and the impact factors $\Phi$ are expressed through

the Reggeon vertices according to Ref. [12]. Note that the appearance of the factors $\left(\hat{\vec{q}}_{1}^{2} \hat{\vec{q}}_{2}^{2}\right)^{-1}$ in (5) and $\left(\vec{q}_{1}^{2} \vec{q}_{2}^{2}\right)^{-1}$ in (6) cannot be explained by a change of the normalization (11). At this 
point we have to recognize that there is a substantial freedom in the definition of the kernel. Indeed, the expression (5) is not changed by the transformation

$$
\hat{\mathcal{K}} \rightarrow \hat{\mathcal{O}}^{-1} \hat{\mathcal{K}} \hat{\mathcal{O}}, \quad\left\langle A^{\prime} \bar{A}\left|\rightarrow\left\langle A^{\prime} \bar{A}|\hat{\mathcal{O}},| \bar{B}^{\prime} B\right\rangle \rightarrow \hat{\mathcal{O}}^{-1}\right| \bar{B}^{\prime} B\right\rangle
$$

with arbitrary nonsingular operator $\hat{\mathcal{O}}$. Actually we use a kernel related with the one defined in Ref. [12] by such transformation with $\hat{\mathcal{O}}=\left(\hat{\vec{q}}_{1}^{2} \hat{\vec{q}}_{2}^{2}\right)^{1 / 2}$. The reason is that in the leading order the kernel which is conformal invariant and is simply related to the dipole kernel is not the kernel defined in (6), but just the kernel (3) [6, 11]. Remind that in this paper we consider scattering of colorless objects and therefore only the color singlet state in the $t$-channel.

In the leading order the trajectory in the momentum representation is given by

$$
\left\langle\vec{q}_{i}\left|\hat{\omega}_{i}\right| \vec{q}_{i}^{\prime}\right\rangle=-\delta\left(\vec{q}_{i}-\vec{q}_{i}^{\prime}\right) \frac{g^{2} N_{c} \vec{q}_{i}^{2}}{2(2 \pi)^{D-1}} \int \frac{d^{D-2} r}{\vec{r}^{2}\left(\vec{q}_{i}-\vec{r}\right)^{2}}=-\delta\left(\vec{q}_{i}-\vec{q}_{i}^{\prime}\right) g^{2} \frac{N_{c} \Gamma(1-\epsilon)}{(4 \pi)^{D / 2}} \frac{\Gamma^{2}(\epsilon)}{\Gamma(2 \epsilon)}\left(\vec{q}_{i}^{2}\right)^{\epsilon}
$$

and the "real" part of the kernel by

$$
\left\langle\overrightarrow{q_{1}}, \overrightarrow{q_{2}}\left|\hat{\mathcal{K}}_{r}\right| \vec{q}_{1}^{\prime}, \vec{q}_{2}^{\prime}\right\rangle=\delta\left(\vec{q}-\vec{q}^{\prime}\right) \frac{g^{2} N_{c}}{(2 \pi)^{D-1}} \frac{1}{\vec{q}_{1}^{2} \vec{q}_{2}^{2}}\left(\frac{\vec{q}_{1}^{2} \vec{q}_{2}^{2}+\vec{q}_{2}^{2} \vec{q}_{1}^{\prime 2}}{\vec{k}^{2}}-\vec{q}^{2}\right) .
$$

Accordingly, in the coordinate representation we obtain

$$
\left\langle\vec{r}_{1}, \vec{r}_{2}\left|\hat{\omega}_{1}+\hat{\omega}_{2}\right| \vec{r}_{1}^{\prime}, \vec{r}_{2}^{\prime}\right\rangle=\frac{g^{2} N_{c} \Gamma^{2}(1+\epsilon)}{8 \pi^{3+2 \epsilon}}\left[\frac{\delta\left(\vec{r}_{1}-\vec{r}_{1}^{\prime}\right)}{\left(\vec{r}_{2}-\vec{r}_{2}^{\prime}\right)^{2(1+2 \epsilon)}}+\frac{\delta\left(\vec{r}_{2}-\vec{r}_{2}^{\prime}\right)}{\left(\vec{r}_{1}-\vec{r}_{1}^{\prime}\right)^{2(1+2 \epsilon)}}\right]
$$

and

$$
\begin{gathered}
\left\langle\vec{r}_{1}, \vec{r}_{2}\left|\hat{\mathcal{K}}_{r}\right| \vec{r}_{1}^{\prime}, \vec{r}_{2}^{\prime}\right\rangle=\frac{g^{2} N_{c} \Gamma^{2}(1+\epsilon)}{4 \pi^{3+2 \epsilon}} \int d^{D-2} \rho \frac{\left(\vec{r}_{1}-\vec{\rho}\right)}{\left(\vec{r}_{1}-\vec{\rho}\right)^{2(1+\epsilon)}} \frac{\left(\vec{r}_{2}-\vec{\rho}\right)}{\left(\vec{r}_{2}-\vec{\rho}\right)^{2(1+\epsilon)}} \\
\times\left(\delta\left(\vec{r}_{1}-\vec{r}_{1}^{\prime}\right)-\delta\left(\vec{r}_{1}^{\prime}-\vec{\rho}\right)\right)\left(\delta\left(\vec{r}_{2}-\vec{r}_{2}^{\prime}\right)-\delta\left(\vec{r}_{2}^{\prime}-\vec{\rho}\right)\right) .
\end{gathered}
$$

Therefore the BFKL kernel can be written as

$$
\begin{gathered}
\left\langle\vec{r}_{1}, \vec{r}_{2}|\hat{\mathcal{K}}| \vec{r}_{1}^{\prime}, \vec{r}_{2}^{\prime}\right\rangle=\left\langle\vec{r}_{1}, \vec{r}_{2}\left|\hat{\mathcal{K}}_{d}\right| \vec{r}_{1}^{\prime}, \vec{r}_{2}^{\prime}\right\rangle \\
-\frac{g^{2} N_{c} \Gamma^{2}(1+\epsilon)}{8 \pi^{3+2 \epsilon}}\left[\frac{\delta\left(\vec{r}_{1}-\vec{r}_{1}^{\prime}\right)}{\left(\vec{r}_{1}-\vec{r}_{2}^{\prime}\right)^{2(1+2 \epsilon)}}+\frac{\delta\left(\vec{r}_{2}-\vec{r}_{2}^{\prime}\right)}{\left(\vec{r}_{2}-\vec{r}_{1}^{\prime}\right)^{2(1+2 \epsilon)}}-2 \frac{\delta\left(\vec{r}_{1}^{\prime}-\vec{r}_{2}^{\prime}\right)\left(\vec{r}_{1}-\vec{r}_{1}^{\prime}\right)\left(\vec{r}_{2}-\vec{r}_{2}^{\prime}\right)}{\left(\vec{r}_{1}-\vec{r}_{1}^{\prime}\right)^{2(1+\epsilon)}\left(\vec{r}_{2}-\vec{r}_{2}^{\prime}\right)^{2(1+\epsilon)}}\right]
\end{gathered}
$$

where

$$
\left\langle\vec{r}_{1}, \vec{r}_{2}\left|\hat{\mathcal{K}}_{d}\right| \vec{r}_{1}^{\prime}, \vec{r}_{2}^{\prime}\right\rangle=\frac{g^{2} N_{c} \Gamma^{2}(1+\epsilon)}{8 \pi^{3+2 \epsilon}} \int d^{2+2 \epsilon} \rho\left(\frac{\left(\vec{r}_{1}-\vec{\rho}\right)}{\left(\vec{r}_{1}-\vec{\rho}\right)^{2(1+\epsilon)}}-\frac{\left(\vec{r}_{2}-\vec{\rho}\right)}{\left(\vec{r}_{2}-\vec{\rho}\right)^{2(1+\epsilon)}}\right)^{2}
$$




$$
\times\left(\delta\left(\vec{r}_{1}-\vec{r}_{1}^{\prime}\right) \delta\left(\vec{r}_{2}^{\prime}-\vec{\rho}\right)+\delta\left(\vec{r}_{2}-\vec{r}_{2}^{\prime}\right) \delta\left(\vec{r}_{1}^{\prime}-\vec{\rho}\right)-\delta\left(\vec{r}_{1}-\vec{r}_{1}^{\prime}\right) \delta\left(\vec{r}_{2}-\vec{r}_{2}^{\prime}\right)\right)
$$

is just the dipole kernel in the $(D-2)$-dimensional space. It can be seen from (14) that the BFKL and dipole kernels are not equivalent. However, if we consider scattering of colorless objects, there is freedom in the definition of the kernel [6, 11]. The matter is that for colorless objects the impact factors $\left\langle A^{\prime} \bar{A}\right|$ in (5) have the property of "gauge invariance": they turn into zero at zero transverse momenta, i.e. $\Phi_{A^{\prime} A}(\overrightarrow{0}, \vec{q})=\Phi_{A^{\prime} A}(\vec{q}, \overrightarrow{0})=0$. This means that $\left\langle A^{\prime} \bar{A} \mid \Psi\right\rangle=0$ for any $|\Psi\rangle$, if $\left\langle\vec{q}_{1}, \vec{q}_{2} \mid \Psi\right\rangle$ contains $\delta\left(\vec{q}_{1}\right)$ or $\delta\left(\vec{q}_{2}\right)$ (or, equivalently, in the coordinate space that $\left\langle\vec{r}_{1}, \vec{r}_{2} \mid \Psi\right\rangle$ does not depend either on $\vec{r}_{1}$ or on $\vec{r}_{2}$ ). As it can be seen from (3), (10) and (11), the BFKL kernel conserves this property, i.e. if $\left\langle A^{\prime} \bar{A}\right|$ is "gauge invariant", then also $\left\langle A^{\prime} \bar{A}\right| \hat{\mathcal{K}}$ is "gauge invariant". This property of the BFKL kernel is ensured by the vanishing of its "real" part $\left\langle\vec{q}_{1}, \vec{q}_{2}\left|\hat{\mathcal{K}}_{r}\right| \vec{q}_{1}^{\prime}, \vec{q}_{2}^{\prime}\right\rangle$ (11) at $\vec{q}_{1}^{\prime}=0$ or $\vec{q}_{2}^{\prime}=0$ and permits to change in (5) the input

$$
|\operatorname{In}\rangle \equiv\left(\hat{\vec{q}}_{1}^{2} \hat{\vec{q}}_{2}^{2}\right)^{-1}\left|\bar{B}^{\prime} B\right\rangle
$$

for $\left|\operatorname{In}_{d}\right\rangle$, where $\left|\operatorname{In}_{d}\right\rangle$ has the "dipole" property $\left\langle\vec{r}, \vec{r} \mid \operatorname{In}_{d}\right\rangle=0$ (in the momentum representation $\left.\int d^{D-2} q_{1} d^{D-2} q_{2}\left\langle\vec{q}_{1}, \vec{q}_{2} \mid \operatorname{In}_{d}\right\rangle=0\right)$. Indeed, this can be done using substitutions of the type

$$
\left\langle\vec{r}_{1}, \vec{r}_{2} \mid \operatorname{In}\right\rangle \rightarrow\left\langle\vec{r}_{1}, \vec{r}_{2} \mid \operatorname{In}\right\rangle-a\left\langle\vec{r}_{1}, \vec{r}_{1} \mid \operatorname{In}\right\rangle-(1-a)\left\langle\vec{r}_{2}, \vec{r}_{2} \mid \operatorname{In}\right\rangle
$$

or, in the momentum representation,

$$
\left\langle\vec{q}_{1}, \vec{q}_{2} \mid \operatorname{In}\right\rangle \rightarrow\left\langle\vec{q}_{1}, \vec{q}_{2} \mid \operatorname{In}\right\rangle-\left[a \delta\left(\vec{q}_{2}\right)+(1-a) \delta\left(\vec{q}_{1}\right)\right] \int d^{D-2} q_{1}^{\prime} d^{D-2} q_{2}^{\prime}\left\langle\vec{q}_{1}^{\prime}, \vec{q}_{2}^{\prime} \mid \operatorname{In}\right\rangle \delta\left(\vec{q}_{1}+q_{2}-\vec{q}_{1}^{\prime}-\vec{q}_{2}^{\prime}\right),
$$

which do not change the matrix element (5). But for inputs of the "dipole" kind the last three terms in the BFKL kernel (14) can be omitted: the first two of them owing to the gauge invariance of $\left\langle A^{\prime} \bar{A}\right| \hat{\mathcal{K}}^{n}$, and the third because of $\hat{\mathcal{K}}_{d}$ conserves the "dipole" property, since $\left\langle\vec{r}_{1}, \vec{r}_{2}\left|\hat{\mathcal{K}}_{d}\right| \vec{r}_{1}^{\prime}, \vec{r}_{2}^{\prime}\right\rangle$ turns into zero at $\vec{r}_{1}=\vec{r}_{2}$ (see (15) ). After that the BFKL kernel turns to the dipole one. Note, however, that it does not mean equivalence of the kernels; in particular, their Green's functions are different. Actually, the BFKL kernel acts in a wider space of functions than the dipole one.

Thus, the BFKL and dipole kernels are not related by a simple Fourier transform. However, by calculating matrix elements of the type (5) with "gauge invariant" impact factors $\left\langle A^{\prime} \bar{A}\right|$, one can change the input (16) into another one, $\left|\operatorname{In}_{d}\right\rangle$, with the "dipole" property $\left\langle\vec{r}, \vec{r} \mid \operatorname{In}_{d}\right\rangle=0$. Then, due to the fact that $\left\langle A^{\prime} \bar{A}\right| \hat{\mathcal{K}}^{n}$ is "gauge invariant" as well as $\left\langle A^{\prime} \bar{A}\right|$, one can add to $\left\langle\vec{r}_{1} \vec{r}_{2}|\hat{\mathcal{K}}| \vec{r}_{1}^{\prime} \vec{r}_{2}^{\prime}\right\rangle$ terms not depending on $\vec{r}_{1}$ and $\vec{r}_{2}$ (in the momentum space proportional to $\delta\left(\vec{q}_{1}\right)$ or $\left.\delta\left(\vec{q}_{2}\right)\right)$. They can be chosen in such a way that after their addition the part of the kernel not containing $\delta\left(\vec{r}_{1}^{\prime}-\vec{r}_{2}^{\prime}\right)$ becomes conserving the "dipole" property. After that the "dipole" property of the $\left|\operatorname{In}_{d}\right\rangle$ permits to omit the terms proportional to $\delta\left(\vec{r}_{1}^{\prime}-\vec{r}_{2}^{\prime}\right)$ (in the momentum 
space depending not separately on $\vec{q}_{1}^{\prime}$ and $\vec{q}_{2}^{\prime}$, but only on the sum $\vec{q}_{1}^{\prime}+\vec{q}_{2}^{\prime}=\vec{q}_{1}+\vec{q}_{2}$ ). In such a way we come from the BFKL to the dipole kernel.

The use of the dipole kernel must not lead neither to infrared, nor to ultraviolet singularities. The kernel $\hat{\mathcal{K}}_{d}$ satisfies this requirement. Indeed, for $\epsilon=0$ we have from (15)

$$
\left\langle\vec{r}_{1}, \vec{r}_{2}\left|\hat{\mathcal{K}}_{d}\right| \Psi\right\rangle=\frac{g^{2} N_{c}}{8 \pi^{3}} \int d^{2} \rho \frac{\left(\vec{r}_{1}-\vec{r}_{2}\right)^{2}}{\left(\vec{r}_{1}-\vec{\rho}\right)^{2}\left(\vec{r}_{2}-\vec{\rho}\right)^{2}}\left(\left\langle\vec{r}_{1}, \vec{\rho} \mid \Psi\right\rangle+\left\langle\vec{\rho}, \vec{r}_{2} \mid \Psi\right\rangle-\left\langle\vec{r}_{1}, \vec{r}_{2} \mid \Psi\right\rangle\right) .
$$

The absence of infrared singularities is evident. Ultraviolet singularities cancel taking into account the "dipole" property $\langle\vec{r}, \vec{r} \mid \Psi\rangle=0$.

The dipole form of the kernel is just the form which exhibits the conformal invariance. Conformal transformations in the two-dimensional space $\vec{r}=(x, y)$ can be written as

$$
z \rightarrow \frac{a z+b}{c z+d}
$$

where $z=x+i y, a, b, c, d$ are complex numbers, with $a d-b c \neq 0$. Under these transformations, one has

$$
\begin{gathered}
z_{1}-z_{2} \rightarrow \frac{z_{1}-z_{2}}{\left(c z_{1}+d\right)\left(c z_{2}+d\right)}(a d-b c), \\
d z d z^{*} \rightarrow d z d z^{*} \frac{|a d-b c|^{2}}{\left|(c z+d)^{2}\right|^{2}},
\end{gathered}
$$

so that the conformal invariance of the dipole kernel is evident from (17).

In the momentum representation the kernels are connected by the relation

$$
\left\langle\overrightarrow{q_{1}} \vec{q}_{2}|\hat{\mathcal{K}}| \vec{q}_{1}^{\prime} \vec{q}_{2}^{\prime}\right\rangle=\left\langle\vec{q}_{1} \vec{q}_{2}\left|\hat{\mathcal{K}}{ }_{d}\right| \vec{q}_{1}^{\prime} \vec{q}_{2}^{\prime}\right\rangle-\delta\left(\vec{q}-\vec{q}^{\prime}\right)\left[\delta\left(\vec{q}_{2}\right) \omega\left(\vec{q}_{2}^{\prime}\right)+\delta\left(\vec{q}_{1}\right) \omega\left(\vec{q}_{1}^{\prime}\right)+\frac{g^{2} N_{c}}{(2 \pi)^{3+2 \epsilon}} \frac{2 \vec{q}_{1} \vec{q}_{2}}{{\overrightarrow{q_{1}}}^{2}{\overrightarrow{q_{2}}}^{2}}\right] .
$$

This equation can be obtained by the direct transformation of (14) in the momentum representation. From (20) and from (3), (10) and (11) it follows that

$$
\int d^{D-2} q_{1} d^{D-2} q_{2}\left\langle\vec{q}_{1} \vec{q}_{2}\left|\hat{\mathcal{K}}_{d}\right| \vec{q}_{1}^{\prime} \vec{q}_{2}^{\prime}\right\rangle=0
$$

which is the expression of the "dipole" property of $\hat{\mathcal{K}}_{d}$ in the momentum space. This means that the dipole kernel does not satisfy to the "bootstrap condition" [11], which can be written as

$$
\int d^{D-2} q_{1} d^{D-2} q_{2}\left\langle\vec{q}_{1} \vec{q}_{2}\left|\left(\hat{\mathcal{K}}+\hat{\omega}_{1}+\hat{\omega}_{2}-2 \omega(t)\right)\right| \vec{q}_{1}^{\prime} \vec{q}_{2}^{\prime}\right\rangle=0 .
$$




\section{The unrenormalized NLO kernel in coordinate repre- sentation}

In this Section, and from now on, we consider only the quark contribution to the NLO kernel. Moreover, we use the large $N_{c}$ limit, where only the "non-Abelian" part of the "real" contribution survives, so that the contribution is strongly simplified [3].

The quark contribution $\omega^{Q}$ to the trajectory appears at the two-loop level only. For the case of $n_{f}$ massless quark flavours we have [3]

$$
\begin{aligned}
\left\langle\vec{q}\left|\hat{\omega}^{Q}\right| \vec{q}^{\prime}\right\rangle=\delta & \left(\vec{q}-\vec{q}^{\prime}\right) \frac{8 g^{4} N_{c} n_{f} \Gamma^{2}(1-\epsilon) \Gamma^{2}(2+\epsilon) \Gamma^{2}(1+\epsilon)}{(4 \pi)^{4+2 \epsilon} \Gamma(4+2 \epsilon) \Gamma(1+2 \epsilon)}\left(\vec{q}^{2}\right)^{2 \epsilon} \frac{1}{\epsilon^{2}} \\
& \times\left(1-\frac{3 \Gamma(1-2 \epsilon) \Gamma^{2}(1+2 \epsilon)}{2 \Gamma^{2}(1-\epsilon) \Gamma(1+\epsilon) \Gamma(1+3 \epsilon)}\right) .
\end{aligned}
$$

In the coordinate representation we get

$$
\left\langle\vec{r}_{1} \vec{r}_{2}\left|\hat{\omega}_{1}^{Q}+\hat{\omega}_{2}^{Q}\right| \vec{r}_{1}^{\prime} \vec{r}_{2}^{\prime}\right\rangle=-a_{v}\left[\frac{\delta\left(\vec{r}_{1}-\vec{r}_{1}^{\prime}\right)}{\epsilon\left(\vec{r}_{2}-\vec{r}_{2}^{\prime}\right)^{2(1+3 \epsilon)}}+\frac{\delta\left(\vec{r}_{2}-\vec{r}_{2}^{\prime}\right)}{\epsilon\left(\vec{r}_{1}-\vec{r}_{1}^{\prime}\right)^{2(1+3 \epsilon)}}\right],
$$

where

$$
\begin{gathered}
a_{v}=2 a_{r}\left(\kappa-\frac{3}{2}\right), \quad \kappa=\frac{\Gamma^{2}(1-\epsilon) \Gamma(1+\epsilon) \Gamma(1+3 \epsilon)}{\Gamma(1-2 \epsilon) \Gamma^{2}(1+2 \epsilon)}, \\
a_{r}=\frac{2 g^{4} N_{c} n_{f} 2^{1+4 \epsilon} \Gamma(1+\epsilon) \Gamma(1+2 \epsilon)}{(4 \pi)^{2+\epsilon}(2 \pi)^{D-1}} \frac{\Gamma^{2}(2+\epsilon)}{\Gamma(4+2 \epsilon)} .
\end{gathered}
$$

The contribution of real $q \bar{q}$ production is given by Eq. (47) of Ref. [3] 1):

$$
\begin{gathered}
\left\langle\vec{q}_{1} \vec{q}_{2}\left|\hat{\mathcal{K}}_{r}^{Q}\right| \vec{q}_{1}^{\prime} \vec{q}_{2}^{\prime}\right\rangle=\delta\left(\vec{q}-\vec{q}^{\prime}\right) \frac{2 g^{4} N_{c} n_{f}}{(4 \pi)^{2+\epsilon}} \frac{\Gamma(1-\epsilon)}{\epsilon(2 \pi)^{D-1}} \frac{\Gamma^{2}(2+\epsilon)}{\Gamma(4+2 \epsilon) \vec{q}_{1}^{2} \vec{q}_{2}^{2}} \\
\times\left\{2 \vec{k}^{2(\epsilon-1)}\left(\vec{q}_{1}^{2}{\vec{q}_{2}^{\prime}}^{2}+\vec{q}_{2}^{2}{\overrightarrow{q_{1}}}^{2}\right)+\vec{q}^{2}\left(2 \vec{q}^{2 \epsilon}-\vec{q}_{1}^{2 \epsilon}-\vec{q}_{1}^{\prime 2 \epsilon}-\vec{q}_{2}^{2 \epsilon}-\vec{q}_{2}^{2 \epsilon}\right)\right. \\
\left.-\frac{\left(\vec{q}_{1}^{2} \vec{q}_{2}^{\prime 2}-\vec{q}_{2}^{2} \vec{q}_{1}^{\prime 2}\right)}{\vec{k}^{2}}\left(\vec{q}_{1}^{2 \epsilon}-\vec{q}_{1}^{\prime 2 \epsilon}-\vec{q}_{2}^{2 \epsilon}+\vec{q}_{2}^{2 \epsilon}\right)\right\} .
\end{gathered}
$$

\footnotetext{
${ }^{1}$ In Eq. (47) of Ref. [3] there is actually a misprint in one sign, easily detectable by comparison with Eq. (48) of that paper or with Eq. (27) of this work, which are correct.
} 
It is easy to see that the expression in the curly brackets vanishes when any of the $\vec{q}_{i}$ 's or $\vec{q}_{i}^{\prime}$ 's tends to zero. Vanishing at $\vec{q}_{i}^{\prime}=0$ is important for us because it means that $\hat{\mathcal{K}}^{Q}$ conserves the "gauge invariance" of the impact factor $\left\langle A^{\prime} \bar{A}\right|$ as well as the kernel in the leading order.

In the coordinate representation $\hat{\mathcal{K}}_{r}^{Q}$ can be presented as (for details, see Ref. [13])

$$
\begin{aligned}
& \left\langle\vec{r}_{1} \vec{r}_{2}\left|\hat{\mathcal{K}}_{r}^{Q}\right| \vec{r}_{1}^{\prime} \vec{r}_{2}^{\prime}\right\rangle=a_{r}\left[\delta\left(\vec{r}_{1}-\vec{r}_{1}^{\prime}\right) \delta\left(\vec{r}_{2}-\vec{r}_{2}^{\prime}\right) \int d^{D-2} \rho f\left(\vec{r}_{1}, \vec{r}_{2} ; \rho\right)+\delta\left(\vec{r}_{1}^{\prime}-\vec{r}_{2}^{\prime}\right) f_{0}\left(\vec{r}_{1}, \vec{r}_{2} ; \vec{r}_{1}^{\prime}\right)\right. \\
& \left.\quad+\delta\left(\vec{r}_{1}-\vec{r}_{1}^{\prime}\right) f_{1}^{r}\left(\vec{r}_{1}, \vec{r}_{2} ; \vec{r}_{2}^{\prime}\right)+\delta\left(\vec{r}_{2}-\vec{r}_{2}^{\prime}\right) f_{2}^{r}\left(\vec{r}_{1}, \vec{r}_{2} ; \vec{r}_{1}^{\prime}\right)+\frac{\Gamma(1+\epsilon)}{\pi^{1+\epsilon}} f\left(\vec{r}_{1}, \vec{r}_{2} ; \vec{r}_{1}^{\prime}, \vec{r}_{2}^{\prime}\right)\right]
\end{aligned}
$$

where

$$
\begin{aligned}
& f\left(\vec{r}_{1}, \vec{r}_{2} ; \vec{\rho}\right)=\frac{\left(\vec{r}_{1}-\vec{\rho}\right)\left(\vec{r}_{2}-\vec{\rho}\right)}{\epsilon\left(\vec{r}_{1}-\vec{\rho}\right)^{2(1+\epsilon)}\left(\vec{r}_{2}-\vec{\rho}\right)^{2(1+\epsilon)}}\left(\frac{1}{\left(\vec{r}_{1}-\vec{\rho}\right)^{2 \epsilon}}+\frac{1}{\left(\vec{r}_{2}-\vec{\rho}\right)^{2 \epsilon}}\right), \\
& f_{0}\left(\vec{r}_{1}, \vec{r}_{2} ; \vec{\rho}\right)=\frac{\left(\vec{r}_{1}-\vec{\rho}\right)\left(\vec{r}_{2}-\vec{\rho}\right)}{\epsilon\left(\vec{r}_{1}-\vec{\rho}\right)^{2(1+\epsilon)}\left(\vec{r}_{2}-\vec{\rho}\right)^{2(1+\epsilon)}}\left(\frac{1}{\left(\vec{r}_{1}-\vec{\rho}\right)^{2 \epsilon}}+\frac{1}{\left(\vec{r}_{2}-\vec{\rho}\right)^{2 \epsilon}}\right) \\
& +\frac{1}{\left(\vec{r}_{1}-\vec{\rho}\right)^{2(1+2 \epsilon)}}\left(\frac{1}{\epsilon\left(\vec{r}_{2}-\vec{\rho}\right)^{2 \epsilon}}-\frac{1}{\epsilon\left(\vec{r}_{1}-\vec{r}_{2}\right)^{2 \epsilon}}\right)+\frac{1}{\left(\vec{r}_{2}-\vec{\rho}\right)^{2(1+2 \epsilon)}}\left(\frac{1}{\epsilon\left(\vec{r}_{1}-\vec{\rho}\right)^{2 \epsilon}}-\frac{1}{\epsilon\left(\vec{r}_{1}-\vec{r}_{2}\right)^{2 \epsilon}}\right) \\
& +2 \int \frac{d^{D-2} \vec{r}_{3}}{\pi^{1+\epsilon}} \frac{\Gamma(1+\epsilon)}{\left(\vec{r}_{3}-\vec{\rho}\right)^{2(1+2 \epsilon)}} \frac{\left(\vec{r}_{1}-\vec{r}_{3}\right)\left(\vec{r}_{2}-\vec{r}_{3}\right)}{\left(\vec{r}_{1}-\vec{r}_{3}\right)^{2(1+\epsilon)}\left(\vec{r}_{2}-\vec{r}_{3}\right)^{2(1+\epsilon)}}, \\
& f_{1}^{r}\left(\vec{r}_{1}, \vec{r}_{2} ; \vec{\rho}\right)=-2 \frac{\left(\vec{r}_{1}-\vec{\rho}\right)\left(\vec{r}_{2}-\vec{\rho}\right)}{\epsilon\left(\vec{r}_{1}-\vec{\rho}\right)^{2(1+2 \epsilon)}\left(\vec{r}_{2}-\vec{\rho}\right)^{2(1+\epsilon)}}-\frac{1}{\epsilon\left(\vec{r}_{1}-\vec{\rho}\right)^{2(1+2 \epsilon)}}\left(\frac{1}{\left(\vec{r}_{2}-\vec{\rho}\right)^{2 \epsilon}}-\frac{1}{\left(\vec{r}_{1}-\vec{r}_{2}\right)^{2 \epsilon}}\right) \\
& -\frac{\left(\vec{r}_{1}-\vec{\rho}\right)\left(\vec{r}_{2}-\vec{\rho}\right)}{\epsilon\left(\vec{r}_{1}-\vec{\rho}\right)^{2(1+\epsilon)}\left(\vec{r}_{2}-\vec{\rho}\right)^{2(1+2 \epsilon)}}-\int \frac{d^{D-2} \vec{r}_{3}}{\pi^{1+\epsilon}} \frac{\Gamma(1+\epsilon)}{\left(\vec{r}_{3}-\vec{\rho}\right)^{2(1+2 \epsilon)}} \frac{\left(\vec{r}_{1}-\vec{r}_{3}\right)\left(\vec{r}_{2}-\vec{r}_{3}\right)}{\left(\vec{r}_{1}-\vec{r}_{3}\right)^{2(1+\epsilon)}\left(\vec{r}_{2}-\vec{r}_{3}\right)^{2(1+\epsilon)}} \\
& f_{2}^{r}\left(\vec{r}_{1}, \vec{r}_{2} ; \vec{\rho}\right)=f_{1}^{r}\left(\vec{r}_{2}, \vec{r}_{1} ; \vec{\rho}\right), \\
& f\left(\vec{r}_{1}, \vec{r}_{2} ; \vec{r}_{1}^{\prime}, \vec{r}_{2}^{\prime}\right)=f\left(\vec{r}_{1}, \vec{r}_{2}\right)+1 \leftrightarrow 2, \\
& f\left(\vec{r}_{1}, \vec{r}_{2}\right)=\frac{1}{\left(\vec{r}_{1}-\vec{r}_{1}^{\prime}\right)^{2(1+2 \epsilon)}} \frac{\left(\vec{r}_{2}-\vec{r}_{2}^{\prime}\right)\left(\vec{r}_{2}^{\prime}-\vec{r}_{1}^{\prime}\right)}{\left(\vec{r}_{2}-\vec{r}_{2}^{\prime}\right)^{2(1+\epsilon)}\left(\vec{r}_{2}^{\prime}-\vec{r}_{1}^{\prime}\right)^{2(1+\epsilon)}} \\
& +\left(\frac{1}{\left(\vec{r}_{1}-\vec{r}_{1}^{\prime}\right)^{2(1+2 \epsilon)}}-\frac{1}{\left(\vec{r}_{1}^{\prime}-\vec{r}_{2}^{\prime}\right)^{2(1+2 \epsilon)}}\right) \frac{\left(\vec{r}_{1}-\vec{r}_{2}^{\prime}\right)\left(\vec{r}_{2}-\vec{r}_{2}^{\prime}\right)}{\left(\vec{r}_{1}-\vec{r}_{2}^{\prime}\right)^{2(1+\epsilon)}\left(\vec{r}_{2}-\vec{r}_{2}^{\prime}\right)^{2(1+\epsilon)}},
\end{aligned}
$$

where $1 \leftrightarrow 2$ means $\vec{r}_{1} \leftrightarrow \vec{r}_{2}, \quad \vec{r}_{1}^{\prime} \leftrightarrow \vec{r}_{2}^{\prime}$. Note, that actually only the integral

$$
\int d^{D-2} \rho f\left(\vec{r}_{1}, \vec{r}_{2} ; \rho\right)=\frac{\pi^{1+\epsilon}}{\epsilon \Gamma(1+\epsilon)\left(\vec{r}_{1}-\vec{r}_{2}\right)^{4 \epsilon}}
$$


is fixed, so that there is a large arbitrariness in definition of $f\left(\vec{r}_{1}, \vec{r}_{2} ; \rho\right)$.

Now the total kernel is presented in the form (28) with the substitution $f_{i}^{r}\left(\vec{r}_{1}, \vec{r}_{2} ; \vec{\rho}\right) \rightarrow$ $f_{i}\left(\vec{r}_{1}, \vec{r}_{2} ; \vec{\rho}\right)$, where

$$
f_{i}\left(\vec{r}_{1}, \vec{r}_{2} ; \vec{\rho}\right)=f_{i}^{r}\left(\vec{r}_{1}, \vec{r}_{2} ; \vec{\rho}\right)-\frac{a_{v}}{a_{r}} \frac{1}{\epsilon\left(\vec{r}_{j}-\vec{\rho}\right)^{2(1+3 \epsilon)}},
$$

with $i=1,2$ and $j \neq i$.

Let us transform the part of the kernel without the term with $\delta\left(\vec{r}_{1}^{\prime}-\vec{r}_{2}^{\prime}\right)$ to the "dipole" form using the freedom, discussed above, to redefine it in matrix elements (5) with "gauge invariant" impact factors $\left\langle A^{\prime} \bar{A}\right|$. As well as in the leading order we change the input (16) for another one, $\left|\operatorname{In}_{d}\right\rangle$, with the "dipole" property $\left\langle\vec{r}, \vec{r} \mid \operatorname{In}_{d}\right\rangle=0$. Then, due to the fact that $\hat{\mathcal{K}}^{Q}$ conserves the "gauge invariance" of the impact factor $\left\langle A^{\prime} \bar{A}\right|$, we add to $\left\langle\vec{r}_{1} \vec{r}_{2}|\hat{\mathcal{K}}| \vec{r}_{1}^{\prime} \vec{r}_{2}^{\prime}\right\rangle$ terms not depending on $\vec{r}_{1}$ and $\vec{r}_{2}$. Moreover, we change $f\left(\vec{r}_{1}, \vec{r}_{2} ; \vec{\rho}\right)$ using the famous property of $(D-2)$-dimensional integrals

$$
\int \frac{d^{D-2} \rho}{\left(\vec{\rho}-\vec{r}_{i}\right)^{\alpha}}=0 .
$$

As a result the considered part must have neither infrared, nor ultraviolet singularities and possess the "dipole" property.

First we note that the functions $f_{i}\left(\vec{r}_{1}, \vec{r}_{2} ; \vec{\rho}\right), i=1,2$, have ultraviolet singularities at $\vec{\rho}=$ $\vec{r}_{j} j \neq i$ (the singularities at $\vec{\rho}=\vec{r}_{i}$ in separate terms cancel each other) which can be removed by adding to $f\left(\vec{r}_{1}, \vec{r}_{2} ; \vec{\rho}\right)$ the term

$$
\frac{a_{v}}{a_{r}}\left(\frac{1}{\epsilon\left(\vec{r}_{1}-\vec{\rho}\right)^{2(1+3 \epsilon)}}+\frac{1}{\epsilon\left(\vec{r}_{2}-\vec{\rho}\right)^{2(1+3 \epsilon)}}\right) .
$$

After that the remaining singularities are infrared. Note that the addition of the term (38) improves also the infrared behavior of $f\left(\vec{r}_{1}, \vec{r}_{2} ; \vec{\rho}\right)$. Since for $\left(\vec{\rho}-\vec{r}_{1}\right)^{2} \simeq\left(\vec{\rho}-\vec{r}_{2}\right)^{2} \gg\left(\vec{r}_{1}-\vec{r}_{2}\right)^{2}$ we have

$$
\begin{aligned}
& \int \frac{d^{D-2} \vec{r}_{3}}{\pi^{1+\epsilon}} \frac{\Gamma(1+\epsilon)}{\left(\vec{r}_{3}-\vec{\rho}\right)^{2(1+2 \epsilon)}} \frac{\left(\vec{r}_{1}-\vec{r}_{3}\right)\left(\vec{r}_{2}-\vec{r}_{3}\right)}{\left(\vec{r}_{1}-\vec{r}_{3}\right)^{2(1+\epsilon)}\left(\vec{r}_{2}-\vec{r}_{3}\right)^{2(1+\epsilon)}} \\
& \simeq \frac{1}{\epsilon\left(\vec{\rho}-\vec{r}_{1}\right)^{2(1+2 \epsilon)}\left(\vec{r}_{1}-\vec{r}_{2}\right)^{2 \epsilon}}-\frac{2 \kappa}{\epsilon\left(\vec{\rho}-\vec{r}_{1}\right)^{2(1+3 \epsilon)}},
\end{aligned}
$$

where $\kappa$ is defined in (25)), the infrared divergences in $f_{i}\left(\vec{r}_{1}, \vec{r}_{2} ; \vec{\rho}\right)$ can be removed by adding $1 /\left[\epsilon\left(\vec{r}_{i}-\vec{\rho}\right)^{2(1+3 \epsilon)}\right]$. Note that this addition does not create ultraviolet singularities due to the assumed "dipole" property of the input. The important fact is that such addition provides the "dipole" property of $f_{i}\left(\vec{r}_{1}, \vec{r}_{2} ; \vec{\rho}\right)$, namely $f_{i}(\vec{r}, \vec{r} ; \vec{\rho})=0$, as it follows immediately using (39)).

Let us turn now to $f\left(\vec{r}_{1}, \vec{r}_{2} ; \vec{r}_{1}^{\prime}, \vec{r}_{2}^{\prime}\right)$. As it can be seen, it has only infrared singularities. We can remove them (not creating new ones) performing the following transformation

$$
f\left(\vec{r}_{1}, \vec{r}_{2}\right) \rightarrow \tilde{f}\left(\vec{r}_{1}, \vec{r}_{2}\right)=f\left(\vec{r}_{1}, \vec{r}_{2}\right)-a f\left(\vec{r}_{1}, \vec{r}_{1}\right)-(1-a) f\left(\vec{r}_{2}, \vec{r}_{2}\right) .
$$


The new function $\tilde{f}\left(\vec{r}_{1}, \vec{r}_{2}\right)$ has the important "dipole" property $\tilde{f}(\vec{r}, \vec{r})=0$, independently from the value of $a$. In our case

$$
\begin{aligned}
& f(\vec{r}, \vec{r})=\frac{1}{\left(\vec{r}-\vec{r}_{1}^{\prime}\right)^{2(1+2 \epsilon)}} \frac{\left(\vec{r}-\vec{r}_{2}^{\prime}\right)\left(\vec{r}_{2}^{\prime}-\vec{r}_{1}^{\prime}\right)}{\left(\vec{r}-\vec{r}_{2}^{\prime}\right)^{2(1+\epsilon)}\left(\vec{r}_{2}^{\prime}-\vec{r}_{1}^{\prime}\right)^{2(1+\epsilon)}} \\
& +\frac{1}{\left(\vec{r}-\vec{r}_{2}^{\prime}\right)^{2(1+2 \epsilon)}}\left[\frac{1}{\left(\vec{r}-\vec{r}_{1}^{\prime}\right)^{2(1+2 \epsilon)}}-\frac{1}{\left(\vec{r}_{1}^{\prime}-\vec{r}_{2}^{\prime}\right)^{2(1+2 \epsilon)}}\right],
\end{aligned}
$$

so that $f\left(\vec{r}_{1}, \vec{r}_{1}\right)$ and $f\left(\vec{r}_{2}, \vec{r}_{2}\right)$ are related by the substitution $1 \leftrightarrow 2$ (that means $\vec{r}_{1} \leftrightarrow \vec{r}_{2}$ and $\left.\vec{r}_{1}^{\prime} \leftrightarrow \vec{r}_{2}^{\prime}\right)$. As a consequence, $f\left(\vec{r}_{1}, \vec{r}_{2} ; \vec{r}_{1}^{\prime}, \vec{r}_{2}^{\prime}\right)$, see (33), does not depend on $a$, so that we can take any $a$. Thus we come to the representation (28), where the term with $\delta\left(\vec{r}_{1}^{\prime}-\vec{r}_{2}^{\prime}\right)$ is omitted and in the other terms we make the replacement $f \rightarrow \tilde{f}$,

$$
\begin{aligned}
& \tilde{f}\left(\vec{r}_{1}, \vec{r}_{2} ; \vec{\rho}\right)=\frac{\left(\vec{r}_{1}-\vec{\rho}\right)\left(\vec{r}_{2}-\vec{\rho}\right)}{\epsilon\left(\vec{r}_{2}-\vec{\rho}\right)^{2(1+\epsilon)}\left(\vec{r}_{1}-\vec{\rho}\right)^{2(1+\epsilon)}}\left(\frac{1}{\left(\vec{r}_{1}-\vec{\rho}\right)^{2 \epsilon}}+\frac{1}{\left(\vec{r}_{2}-\vec{\rho}\right)^{2 \epsilon}}\right) \\
& +\frac{a_{v}}{a_{r}}\left(\frac{1}{\epsilon\left(\vec{r}_{1}-\vec{\rho}\right)^{2(1+3 \epsilon)}}+\frac{1}{\epsilon\left(\vec{r}_{2}-\vec{\rho}\right)^{2(1+3 \epsilon)}}\right), \\
& \tilde{f}_{1}\left(\vec{r}_{1}, \vec{r}_{2} ; \vec{\rho}\right)=-2 \frac{\left(\vec{r}_{1}-\vec{\rho}\right)\left(\vec{r}_{2}-\vec{\rho}\right)}{\epsilon\left(\vec{r}_{1}-\vec{\rho}\right)^{2(1+2 \epsilon)}\left(\vec{r}_{2}-\vec{\rho}\right)^{2(1+\epsilon)}}-\frac{a_{v}}{a_{r}} \frac{1}{\epsilon\left(\vec{r}_{2}-\vec{\rho}\right)^{2(1+3 \epsilon)}}+\frac{1}{\epsilon\left(\vec{r}_{1}-\vec{\rho}\right)^{2(1+3 \epsilon)}} \\
& -\frac{1}{\epsilon\left(\vec{r}_{1}-\vec{\rho}\right)^{2(1+2 \epsilon)}}\left(\frac{1}{\left(\vec{r}_{2}-\vec{\rho}\right)^{2 \epsilon}}-\frac{1}{\left(\vec{r}_{1}-\vec{r}_{2}\right)^{2 \epsilon}}\right)-\frac{\left(\vec{r}_{1}-\vec{\rho}\right)\left(\vec{r}_{2}-\vec{\rho}\right)}{\epsilon\left(\vec{r}_{1}-\vec{\rho}\right)^{2(1+\epsilon)}\left(\vec{r}_{2}-\vec{\rho}\right)^{2(1+2 \epsilon)}} \\
& -\int \frac{d^{D-2} \vec{r}_{3}}{\pi^{1+\epsilon}} \frac{\Gamma(1+\epsilon)}{\left(\vec{r}_{3}-\vec{\rho}\right)^{2(1+2 \epsilon)}} \frac{\left(\vec{r}_{1}-\vec{r}_{3}\right)\left(\vec{r}_{2}-\vec{r}_{3}\right)}{\left(\vec{r}_{1}-\vec{r}_{3}\right)^{2(1+\epsilon)}\left(\vec{r}_{2}-\vec{r}_{3}\right)^{2(1+\epsilon)}} \\
& \tilde{f}_{2}\left(\vec{r}_{1}, \vec{r}_{2} ; \vec{\rho}\right)=\tilde{f}_{1}\left(\vec{r}_{2}, \vec{r}_{1} ; \vec{\rho}\right), \\
& \tilde{f}\left(\vec{r}_{1}, \vec{r}_{2} ; \vec{r}_{1}^{\prime}, \vec{r}_{2}^{\prime}\right)=\tilde{f}\left(\vec{r}_{1}, \vec{r}_{2}\right)+1 \leftrightarrow 2, \\
& \tilde{f}\left(\vec{r}_{1}, \vec{r}_{2}\right)=f\left(\vec{r}_{1}, \vec{r}_{2}\right)-f\left(\vec{r}_{1}, \vec{r}_{1}\right),
\end{aligned}
$$

where $f\left(\vec{r}_{1}, \vec{r}_{2}\right)$ is defined in (34).

\section{Renormalization of the NLO kernel in coordinate rep- resentation}

In order to get the renormalized quark contribution to NLO kernel, we have to express the bare coupling $g$ in terms of the renormalized one $g_{\mu}$ and to add the part proportional to $n_{f}$ coming 
from the coupling renormalization in the leading order kernel (15). In the $\overline{\mathrm{MS}}$ scheme we have

$$
g^{2}=g_{\mu}^{2} \mu^{-2 \epsilon}\left[1+\left(\frac{11}{3}-\frac{2}{3} \frac{n_{f}}{N_{c}}\right) \frac{\bar{g}_{\mu}^{2}}{\epsilon}\right]
$$

where

$$
\bar{g}_{\mu}^{2}=\frac{g_{\mu}^{2} N_{c} \Gamma(1-\epsilon)}{(4 \pi)^{2+\epsilon}} .
$$

Therefore the contribution coming from the coupling renormalization in the leading order kernel is obtained from (15) by the substitution

$$
\frac{g^{2} N_{c} \Gamma^{2}(1+\epsilon)}{8 \pi^{3+2 \epsilon}} \rightarrow-\frac{\bar{g}_{\mu}^{4} n_{f}}{N_{c}} \frac{2^{2+2 \epsilon} \mu^{-2 \epsilon}}{\pi^{1+\epsilon}} \frac{\Gamma^{2}(1+\epsilon)}{3 \epsilon \Gamma(1-\epsilon)}=-\lambda \frac{a_{r}}{\epsilon},
$$

where

$$
\lambda=\frac{\Gamma(1-\epsilon) \Gamma(1+\epsilon) \Gamma(4+2 \epsilon)}{6 \cdot 2^{2 \epsilon} \mu^{-2 \epsilon} \Gamma(1+2 \epsilon) \Gamma^{2}(2+\epsilon)},
$$

and $a_{r}$ is expressed in terms of $\bar{g}_{\mu}$,

$$
a_{r}=\frac{8 \bar{g}_{\mu}^{4} \mu^{-4 \epsilon} 2^{4 \epsilon} \Gamma^{2}(2+\epsilon) \Gamma(1+\epsilon) \Gamma(1+2 \epsilon) n_{f}}{\pi^{1+\epsilon} \Gamma(4+2 \epsilon) \Gamma^{2}(1-\epsilon) N_{c}} .
$$

Then the quark contribution to the renormalized kernel in the "dipole" form is written as

$$
\begin{gathered}
\left\langle\vec{r}_{1} \vec{r}_{2}\left|\hat{\mathcal{K}}_{d}^{Q}\right| \vec{r}_{1}^{\prime} \vec{r}_{2}^{\prime}\right\rangle=a_{r}\left[\delta\left(\vec{r}_{1}-\vec{r}_{1}^{\prime}\right) \delta\left(\vec{r}_{2}-\vec{r}_{2}^{\prime}\right) \int d^{D-2} \rho \bar{f}\left(\vec{r}_{1}, \vec{r}_{2} ; \rho\right)\right. \\
\left.+\delta\left(\vec{r}_{1}-\vec{r}_{1}^{\prime}\right) \vec{f}_{1}\left(\vec{r}_{1}, \vec{r}_{2} ; \vec{r}_{2}^{\prime}\right)+\delta\left(\vec{r}_{2}-\vec{r}_{2}^{\prime}\right) \bar{f}_{2}\left(\vec{r}_{1}, \vec{r}_{2} ; \vec{r}_{1}^{\prime}\right)+\frac{\Gamma(1+\epsilon)}{\pi^{1+\epsilon}} \bar{f}\left(\vec{r}_{1}, \vec{r}_{2} ; \vec{r}_{1}^{\prime}, \vec{r}_{2}^{\prime}\right)\right]
\end{gathered}
$$

where

$$
\begin{gathered}
\bar{f}\left(\vec{r}_{1}, \vec{r}_{2} ; \vec{\rho}\right)=\frac{\left(\vec{r}_{1}-\vec{\rho}\right)\left(\vec{r}_{2}-\vec{\rho}\right)}{\epsilon\left(\vec{r}_{2}-\vec{\rho}\right)^{2(1+\epsilon)}\left(\vec{r}_{1}-\vec{\rho}\right)^{2(1+\epsilon)}}\left(\frac{1}{\left(\vec{r}_{1}-\vec{\rho}\right)^{2 \epsilon}}+\frac{1}{\left(\vec{r}_{2}-\vec{\rho}\right)^{2 \epsilon}}-2 \lambda\right) \\
\left.+\left(\frac{a_{v}}{a_{r}\left(\vec{r}_{1}-\vec{\rho}\right)^{2 \epsilon}}+\lambda\right) \frac{1}{\epsilon\left(\vec{r}_{1}-\vec{\rho}\right)^{2(1+2 \epsilon)}}+\left(\frac{a_{v}}{a_{r}\left(\vec{r}_{2}-\vec{\rho}\right)^{2 \epsilon}}+\lambda\right) \frac{1}{\epsilon\left(\vec{r}_{2}-\vec{\rho}\right)^{2(1+2 \epsilon)}}\right), \\
\quad \bar{f}_{1}\left(\vec{r}_{1}, \vec{r}_{2} ; \vec{\rho}\right)=-2\left(\frac{1}{\left(\vec{r}_{1}-\vec{\rho}\right)^{2 \epsilon}}-\lambda\right) \frac{\left(\vec{r}_{1}-\vec{\rho}\right)\left(\vec{r}_{2}-\vec{\rho}\right)}{\epsilon\left(\vec{r}_{1}-\vec{\rho}\right)^{2(1+\epsilon)}\left(\vec{r}_{2}-\vec{\rho}\right)^{2(1+\epsilon)}} \\
-\left(\frac{a_{v}}{a_{r}\left(\vec{r}_{2}-\vec{\rho}\right)^{2 \epsilon}}+\lambda\right) \frac{1}{\epsilon\left(\vec{r}_{2}-\vec{\rho}\right)^{2(1+2 \epsilon)}}+\left(\frac{1}{\left(\vec{r}_{1}-\vec{\rho}\right)^{2 \epsilon}}-\lambda\right) \frac{1}{\epsilon\left(\vec{r}_{1}-\vec{\rho}\right)^{2(1+2 \epsilon)}}
\end{gathered}
$$




$$
\begin{gathered}
-\frac{1}{\epsilon\left(\vec{r}_{1}-\vec{\rho}\right)^{2(1+2 \epsilon)}}\left(\frac{1}{\left(\vec{r}_{2}-\vec{\rho}\right)^{2 \epsilon}}-\frac{1}{\left(\vec{r}_{1}-\vec{r}_{2}\right)^{2 \epsilon}}\right)-\frac{\left(\vec{r}_{1}-\vec{\rho}\right)\left(\vec{r}_{2}-\vec{\rho}\right)}{\epsilon\left(\vec{r}_{1}-\vec{\rho}\right)^{2(1+\epsilon)}\left(\vec{r}_{2}-\vec{\rho}\right)^{2(1+2 \epsilon)}} \\
-\int \frac{d^{D-2} \vec{r}_{3}}{\pi^{1+\epsilon}} \frac{\Gamma(1+\epsilon)}{\left(\vec{r}_{3}-\vec{\rho}\right)^{2(1+2 \epsilon)}} \frac{\left(\vec{r}_{1}-\vec{r}_{3}\right)\left(\vec{r}_{2}-\vec{r}_{3}\right)}{\left(\vec{r}_{1}-\vec{r}_{3}\right)^{2(1+\epsilon)}\left(\vec{r}_{2}-\vec{r}_{3}\right)^{2(1+\epsilon)}} \\
\vec{f}_{2}\left(\vec{r}_{1}, \vec{r}_{2} ; \vec{\rho}\right)=\vec{f}_{1}\left(\vec{r}_{2}, \vec{r}_{1} ; \vec{\rho}\right), \\
\bar{f}\left(\vec{r}_{1}, \vec{r}_{2} ; \vec{r}_{1}^{\prime}, \vec{r}_{2}^{\prime}\right)=\tilde{f}\left(\vec{r}_{1}, \vec{r}_{2}\right)+1 \leftrightarrow 2, \\
\tilde{f}\left(\vec{r}_{1}, \vec{r}_{2}\right)=f\left(\vec{r}_{1}, \vec{r}_{2}\right)-f\left(\vec{r}_{1}, \vec{r}_{1}\right),
\end{gathered}
$$

and $f\left(\vec{r}_{1}, \vec{r}_{2}\right)$ is defined in (34). These equations give the quark contribution to the dipole kernel at arbitrary $D$.

The limit $\epsilon \rightarrow 0$ can be easily taken. We can put $\epsilon=0$ in the common coefficient $a_{r}$ and in the ratio $a_{r} / a_{v}$ (see (150), (47) and (25)), getting

$$
a_{r}=\frac{\alpha_{s}^{2}(\mu) N_{c} n_{f}}{12 \pi^{3}}, \quad \frac{a_{r}}{a_{v}}=-1
$$

and then we can expand the integrand in $\epsilon$. Using

$$
\begin{gathered}
\int \frac{d^{D-2} \rho}{\pi^{1+\epsilon}} \frac{\Gamma(1+\epsilon)}{\left(\vec{r}_{1}^{\prime}-\vec{\rho}\right)^{2(1+2 \epsilon)}} \frac{\left(\vec{r}_{1}-\vec{\rho}\right)\left(\vec{r}_{2}-\vec{\rho}\right)}{\left(\vec{r}_{1}-\vec{\rho}\right)^{2(1+\epsilon)}\left(\vec{r}_{2}-\vec{\rho}\right)^{2(1+\epsilon)}} \\
\simeq \frac{\left(\vec{r}_{1}-\vec{r}_{1}^{\prime}\right)\left(\vec{r}_{2}-\vec{r}_{1}^{\prime}\right)}{\left(\vec{r}_{1}-\vec{r}_{1}^{\prime}\right)^{2}\left(\vec{r}_{2}-\vec{r}_{1}^{\prime}\right)^{2}}\left(-\frac{1}{\epsilon}+\ln \left(\frac{\left(\vec{r}_{1}-\vec{r}_{1}^{\prime}\right)^{4}\left(\vec{r}_{2}-\vec{r}_{1}^{\prime}\right)^{4}}{\left(\vec{r}_{1}-\vec{r}_{2}\right)^{2}}\right)\right),
\end{gathered}
$$

and

$$
\lambda \simeq 1+\epsilon\left(\frac{5}{3}-2 \psi(1)+\ln \left(\frac{\mu^{2}}{4}\right)\right) \equiv 1-\epsilon \ln \vec{r}_{\mu}^{2} ; \ln \vec{r}_{\mu}^{2}=-\frac{5}{3}+2 \psi(1)-\ln \left(\frac{\mu^{2}}{4}\right)
$$

we obtain finally

$$
\begin{aligned}
& \bar{f}\left(\vec{r}_{1}, \vec{r}_{2} ; \vec{\rho}\right)=\frac{1}{2}\left[-\frac{\left(\vec{r}_{1}-\vec{r}_{2}\right)^{2}}{\left(\vec{r}_{1}-\vec{\rho}\right)^{2}\left(\vec{r}_{2}-\vec{\rho}\right)^{2}} \ln \left(\frac{\vec{r}_{\mu}^{4}}{\left(\vec{r}_{1}-\vec{\rho}\right)^{2}\left(\vec{r}_{2}-\vec{\rho}\right)^{2}}\right)\right. \\
&+\left.\frac{\left(\vec{r}_{2}-\vec{\rho}\right)^{2}-\left(\vec{r}_{1}-\vec{\rho}\right)^{2}}{\left(\vec{r}_{1}-\vec{\rho}\right)^{2}\left(\vec{r}_{2}-\vec{\rho}\right)^{2}} \ln \left(\frac{\left(\vec{r}_{1}-\vec{\rho}\right)^{2}}{\left(\vec{r}_{2}-\vec{\rho}\right)^{2}}\right]\right), \\
& \bar{f}_{1}\left(\vec{r}_{1}, \vec{r}_{2} ; \vec{\rho}\right)=\frac{1}{2}\left[\frac{\left(\vec{r}_{1}-\vec{r}_{2}\right)^{2}}{\left(\vec{r}_{1}-\vec{\rho}\right)^{2}\left(\vec{r}_{2}-\vec{\rho}\right)^{2}} \ln \left(\frac{\vec{r}_{\mu}^{4}}{\left(\vec{r}_{1}-\vec{\rho}\right)^{2}\left(\vec{r}_{1}-\vec{r}_{2}\right)^{2}}\right)\right.
\end{aligned}
$$




$$
\begin{gathered}
\left.+\frac{\left(\vec{r}_{1}-\vec{\rho}\right)^{2}-\left(\vec{r}_{2}-\vec{\rho}\right)^{2}}{\left(\vec{r}_{1}-\vec{\rho}\right)^{2}\left(\vec{r}_{2}-\vec{\rho}\right)^{2}} \ln \left(\frac{\left(\vec{r}_{1}-\vec{\rho}\right)^{2}\left(\vec{r}_{1}-\vec{r}_{2}\right)^{2}}{\left(\vec{r}_{2}-\vec{\rho}\right)^{4}}\right)\right] \\
\vec{f}_{2}\left(\vec{r}_{1}, \vec{r}_{2} ; \vec{\rho}\right)=\vec{f}_{1}\left(\vec{r}_{2}, \vec{r}_{1} ; \vec{\rho}\right) \\
\bar{f}\left(\vec{r}_{1}, \vec{r}_{2} ; \vec{r}_{1}^{\prime}, \vec{r}_{2}^{\prime}\right)=\frac{\left(\vec{r}_{1}-\vec{r}_{2}\right)\left(\vec{r}_{1}^{\prime}-\vec{r}_{2}^{\prime}\right)}{\left(\vec{r}_{1}-\vec{r}_{1}^{\prime}\right)^{2}\left(\vec{r}_{2}-\vec{r}_{2}^{\prime}\right)^{2}\left(\vec{r}_{1}^{\prime}-\vec{r}_{2}^{\prime}\right)^{2}}+\frac{\left(\vec{r}_{1}-\vec{r}_{2}\right)^{2}}{2\left(\vec{r}_{1}-\vec{r}_{2}^{\prime}\right)^{2}\left(\vec{r}_{2}-\vec{r}_{2}^{\prime}\right)^{2}}\left(\frac{1}{\left(\vec{r}_{1}^{\prime}-\vec{r}_{2}^{\prime}\right)^{2}}-\frac{1}{\left(\vec{r}_{1}-\vec{r}_{1}^{\prime}\right)^{2}}\right) \\
+\frac{\left(\vec{r}_{1}-\vec{r}_{2}\right)^{2}}{2\left(\vec{r}_{2}-\vec{r}_{1}^{\prime}\right)^{2}\left(\vec{r}_{1}-\vec{r}_{1}^{\prime}\right)^{2}}\left(\frac{1}{\left(\vec{r}_{1}^{\prime}-\vec{r}_{2}^{\prime}\right)^{2}}-\frac{1}{\left(\vec{r}_{2}-\vec{r}_{2}^{\prime}\right)^{2}}\right) .
\end{gathered}
$$

We notice that the conformal invariance is violated not only by the renormalization. We see also that the result of transformation to the coordinate representation of the BFKL kernel defined in the momentum representation by (3), (4) and (6) does not coincide with the result obtained recently in Ref. [14] by direct calculation of the quark contribution to the dipole kernel in the coordinate representation. This is evident from the presence of the function $\bar{f}\left(\vec{r}_{1}, \vec{r}_{2} ; \vec{r}_{1}^{\prime}, \vec{r}_{2}^{\prime}\right)$, which is absent at large $N_{c}$ in the result of Ref. [14]. However, at this point we have to remind about the freedom in the definition of the BFKL kernel, which was discussed at the beginning of Section 2. We have fixed the operator $\hat{\mathcal{O}}$ in $(9)$, but transformations with $\hat{\mathcal{O}}=1-\hat{O}$, where $\hat{O} \sim g^{2}$, are still possible. At the NLO after such transformation we get

$$
\left\langle\vec{r}_{1} \vec{r}_{2}|\hat{\mathcal{K}}| \vec{r}_{1}^{\prime} \vec{r}_{2}^{\prime}\right\rangle \rightarrow\left\langle\vec{r}_{1} \vec{r}_{2}|\hat{\mathcal{K}}| \vec{r}_{1}^{\prime} \vec{r}_{2}^{\prime}\right\rangle-\left\langle\vec{r}_{1} \vec{r}_{2}\left|\left[\hat{\mathcal{K}}^{(B)}, \hat{O}\right]\right| \vec{r}_{1}^{\prime} \vec{r}_{2}^{\prime}\right\rangle
$$

where $\hat{\mathcal{K}}^{(B)}$ is the leading order kernel.

If we take

$$
\hat{O}=\frac{2 g^{2} n_{f}}{(4 \pi)^{2+\epsilon}} \frac{\Gamma(1-\epsilon) \Gamma^{2}(2+\epsilon)}{\epsilon \Gamma(4+2 \epsilon)}\left(\hat{\vec{q}}_{1}^{2 \epsilon}+\hat{\vec{q}}_{2}^{2 \epsilon}\right),
$$

then, as a result of the transformation, all the functions $f$ in (28) change to $f \rightarrow f+h$, where

$$
\begin{gathered}
h\left(\vec{r}_{1}, \vec{r}_{2} ; \vec{\rho}\right)=0 \\
h_{0}\left(\vec{r}_{1}, \vec{r}_{2} ; \vec{\rho}\right)=\frac{\left(\vec{r}_{1}-\vec{\rho}\right)\left(\vec{r}_{2}-\vec{\rho}\right)}{\epsilon\left(\vec{r}_{1}-\vec{\rho}\right)^{2(1+\epsilon)}\left(\vec{r}_{2}-\vec{\rho}\right)^{2(1+\epsilon)}}\left(\frac{1}{\left(\vec{r}_{1}-\vec{\rho}\right)^{2 \epsilon}}+\frac{1}{\left(\vec{r}_{2}-\vec{\rho}\right)^{2 \epsilon}}\right) \\
h_{1}^{r}\left(\vec{r}_{1}, \vec{r}_{2} ; \vec{\rho}\right)=-\frac{\left(\vec{r}_{1}-\vec{\rho}\right)\left(\vec{r}_{2}-\vec{\rho}\right)}{\epsilon\left(\vec{r}_{1}-\vec{\rho}\right)^{2(1+\epsilon)}\left(\vec{r}_{2}-\vec{\rho}\right)^{2(1+2 \epsilon)}}-\frac{1}{\epsilon\left(\vec{r}_{2}-\vec{\rho}\right)^{2(1+2 \epsilon)}}\left(\frac{1}{\left(\vec{r}_{1}-\vec{\rho}\right)^{2 \epsilon}}-\frac{1}{\left(\vec{r}_{1}-\vec{r}_{2}\right)^{2 \epsilon}}\right) \\
-\int \frac{d^{D-2} \vec{r}_{3}}{\pi^{1+\epsilon}} \frac{\Gamma(1+\epsilon)}{\left(\vec{r}_{3}-\vec{\rho}\right)^{2(1+2 \epsilon)}} \frac{\left(\vec{r}_{1}-\vec{r}_{3}\right)\left(\vec{r}_{2}-\vec{r}_{3}\right)}{\left(\vec{r}_{1}-\vec{r}_{3}\right)^{2(1+\epsilon)}\left(\vec{r}_{2}-\vec{r}_{3}\right)^{2(1+\epsilon)}} \\
h_{2}^{r}\left(\vec{r}_{1}, \vec{r}_{2} ; \vec{\rho}\right)=h_{1}^{r}\left(\vec{r}_{2}, \vec{r}_{1} ; \vec{\rho}\right), \\
h\left(\vec{r}_{1}, \vec{r}_{2} ; \vec{r}_{1}^{\prime}, \vec{r}_{2}^{\prime}\right)=-f\left(\vec{r}_{1}, \vec{r}_{2} ; \vec{r}_{1}^{\prime}, \vec{r}_{2}^{\prime}\right) .
\end{gathered}
$$


Note that the functions $h_{i}^{r}$ have neither ultraviolet nor infrared singularities and possess the dipole property. Taking into account that in the limit $\epsilon \rightarrow 0$ we have

$$
\begin{gathered}
h_{1}^{r}\left(\vec{r}_{2}, \vec{r}_{1} ; \vec{\rho}\right)=\frac{1}{2} \frac{\left(\vec{r}_{1}-\vec{r}_{2}\right)^{2}+\left(\vec{r}_{1}-\vec{\rho}\right)^{2}-\left(\vec{r}_{2}-\vec{\rho}\right)^{2}}{\left(\vec{r}_{1}-\vec{\rho}\right)^{2}\left(\vec{r}_{2}-\vec{\rho}\right)^{2}} \ln \left(\frac{\left(\vec{r}_{1}-\vec{\rho}\right)^{2}}{\left(\vec{r}_{1}-\vec{r}_{2}\right)^{2}}\right), \\
h_{2}^{r}\left(\vec{r}_{1}, \vec{r}_{2} ; \vec{\rho}\right)=h_{1}^{r}\left(\vec{r}_{2}, \vec{r}_{1} ; \vec{\rho}\right),
\end{gathered}
$$

we come to the conclusion that the transformation (62) with $\hat{O}$ defined in (63) leads to the substitutions in (58)-

$$
\bar{f}\left(\vec{r}_{1}, \vec{r}_{2} ; \vec{r}_{1}^{\prime}, \vec{r}_{2}^{\prime}\right) \rightarrow 0, \quad \bar{f}_{1,2}\left(\vec{r}_{1}, \vec{r}_{2} ; \vec{\rho}\right) \rightarrow f_{b}\left(\vec{r}_{1}, \vec{r}_{2} ; \vec{\rho}\right)
$$

where

$$
f_{b}\left(\vec{r}_{1}, \vec{r}_{2} ; \vec{\rho}\right)=\left[\frac{\left(\vec{r}_{1}-\vec{r}_{2}\right)^{2}}{\left(\vec{r}_{1}-\vec{\rho}\right)^{2}\left(\vec{r}_{2}-\vec{\rho}\right)^{2}} \ln \left(\frac{\vec{r}_{\mu}^{2}}{\left(\vec{r}_{1}-\vec{r}_{2}\right)^{2}}\right)+\frac{\left(\vec{r}_{1}-\vec{\rho}\right)^{2}-\left(\vec{r}_{2}-\vec{\rho}\right)^{2}}{\left(\vec{r}_{1}-\vec{\rho}\right)^{2}\left(\vec{r}_{2}-\vec{\rho}\right)^{2}} \ln \left(\frac{\left(\vec{r}_{1}-\vec{\rho}\right)^{2}}{\left(\vec{r}_{2}-\vec{\rho}\right)^{2}}\right)\right] .
$$

Up to the definition of the renormalization scale $\vec{r}_{\mu}^{2}$ (which also can be considered as the result of an appropriate transformation) this function coincides with the one which appears in the result of Ref. [14]. As for $\bar{f}\left(\vec{r}_{2}, \vec{r}_{1} ; \vec{\rho}\right)$, we remind that only the integral (35) is really defined. Without change of the integral we can add to $f\left(\vec{r}_{2}, \vec{r}_{1} ; \vec{\rho}\right)$ in $(29)$ the function $h\left(\vec{r}_{2}, \vec{r}_{1} ; \vec{\rho}\right)$

$$
\begin{gathered}
h\left(\vec{r}_{1}, \overrightarrow{r_{2}} ; \vec{\rho}\right)=\frac{\left(\vec{r}_{1}-\vec{\rho}\right)\left(\vec{r}_{2}-\vec{\rho}\right)}{\epsilon\left(\vec{r}_{1}-\vec{\rho}\right)^{2(1+\epsilon)}\left(\vec{r}_{2}-\vec{\rho}\right)^{2(1+\epsilon)}}\left(\frac{2}{\left(\vec{r}_{1}-\overrightarrow{r_{2}}\right)^{2 \epsilon}}-\frac{1}{\left(\vec{r}_{1}-\vec{\rho}\right)^{2 \epsilon}}-\frac{1}{\left(\vec{r}_{2}-\vec{\rho}\right)^{2 \epsilon}}\right) \\
-\frac{1}{\epsilon\left(\vec{r}_{2}-\vec{\rho}\right)^{2(1+2 \epsilon)}}\left(\frac{1}{\left(\vec{r}_{1}-\vec{r}_{2}\right)^{2 \epsilon}}-\frac{1}{\left(\vec{r}_{1}-\vec{\rho}\right)^{2 \epsilon}}\right)-\frac{1}{\epsilon\left(\vec{r}_{1}-\vec{\rho}\right)^{2(1+2 \epsilon)}}\left(\frac{1}{\left(\vec{r}_{1}-\vec{r}_{2}\right)^{2 \epsilon}}-\frac{1}{\left(\vec{r}_{2}-\vec{\rho}\right)^{2 \epsilon}}\right),
\end{gathered}
$$

with the properties

$$
\begin{gathered}
\int d^{2+2 \epsilon} \rho h\left(\vec{r}_{1}, \vec{r}_{2} ; \vec{\rho}\right)=0, \\
\left.h\left(\vec{r}_{1}, \vec{r}_{2} ; \vec{\rho}\right)\right|_{\epsilon \rightarrow 0}=\frac{1}{2}\left[\frac{\left(\vec{r}_{1}-\vec{r}_{2}\right)^{2}}{\left(\vec{r}_{1}-\vec{\rho}\right)^{2}\left(\vec{r}_{2}-\vec{\rho}\right)^{2}} \ln \left(\frac{\left(\vec{r}_{1}-\vec{r}_{2}\right)^{4}}{\left(\vec{r}_{1}-\vec{\rho}\right)^{2}\left(\vec{r}_{2}-\vec{\rho}\right)^{2}}\right)\right. \\
\left.+\frac{\left(\vec{r}_{2}-\vec{\rho}\right)^{2}-\left(\vec{r}_{1}-\vec{\rho}\right)^{2}}{\left(\vec{r}_{1}-\vec{\rho}\right)^{2}\left(\vec{r}_{2}-\vec{\rho}\right)^{2}} \ln \left(\frac{\left(\vec{r}_{1}-\vec{\rho}\right)^{2}}{\left(\vec{r}_{2}-\vec{\rho}\right)^{2}}\right)\right] .
\end{gathered}
$$

As a result we have in (58) that $\bar{f}\left(\vec{r}_{1}, \vec{r}_{2} ; \vec{\rho}\right) \rightarrow-f_{b}\left(\vec{r}_{1}, \vec{r}_{2} ; \vec{\rho}\right)$. After this the dipole form of the kernel (62), (63) coincides with the result of Ref. [14] (up to the definition of the renormalization scale). 


\section{Direct transformation of the renormalized NLO kernel at $\mathrm{D}=4$}

Till now, in order to be as general and rigorous as possible, we worked starting with the BFKL kernel in the momentum representation at arbitrary space-time dimension $D$. However, the results of transformation to the coordinate representation in the physical space-time dimension $D=4$ can be obtained in a much easier way, if we start from the renormalized BFKL kernel at $D=4$ in a specific form. To obtain this form let us use the renormalized quark contribution to the BFKL kernel in the form [3],

$$
\begin{aligned}
& \left\langle\vec{q}_{1} \vec{q}_{2}\left|\hat{\mathcal{K}}_{r}^{Q}\right| \vec{q}_{1}^{\prime} \vec{q}_{2}^{\prime}\right\rangle_{\text {renorm }}=\delta\left(\vec{q}-\vec{q}^{\prime}\right) F_{r}\left(\vec{q}_{1}, \vec{q}_{1}^{\prime} ; \vec{q}\right), \\
& F_{r}\left(\vec{q}_{1}, \vec{q}_{1}^{\prime} ; \vec{q}\right)=\frac{\bar{q}_{\mu}^{4} \mu^{-2 \epsilon}}{\pi^{1+\epsilon} \Gamma(1-\epsilon)} \frac{2 n_{f}}{3 N_{c}}\left\{\frac{2}{\epsilon}\left(\frac{6[\Gamma(2+\epsilon)]^{2}}{\Gamma(4+2 \epsilon)}\left(\frac{\vec{k}^{2}}{\mu^{2}}\right)^{\epsilon}-1\right)\left(\frac{\vec{q}_{2}^{\prime 2}}{\vec{q}_{2}^{2} \vec{k}^{2}}+\frac{\vec{q}_{1}^{2}}{\vec{q}_{1}^{2} \vec{k}^{2}}-\frac{\vec{q}^{2}}{\vec{q}_{1}^{2} \vec{q}_{2}^{2}}\right)\right. \\
& \left.+\frac{\vec{q}^{2}}{\vec{q}_{1}^{2} \vec{q}_{2}^{2}} \ln \left(\frac{\vec{q}^{4} \vec{k}^{4}}{\vec{q}_{1}^{2} \vec{q}_{2}^{2} \vec{q}_{1}^{\prime 2} \vec{q}_{2}^{\prime 2}}\right)-\left(\frac{\vec{q}_{2}^{\prime 2}}{\vec{q}_{2}^{2} \vec{k}^{2}}-\frac{\vec{q}_{1}^{\prime 2}}{\vec{q}_{1}^{2} \vec{k}^{2}}\right) \ln \left(\frac{\vec{q}_{1}^{2} \vec{q}_{2}^{\prime 2}}{\vec{q}_{2}^{2} \vec{q}_{1}^{\prime 2}}\right)\right\} \text {. }
\end{aligned}
$$

We also use the integral representation for the quark part of the trajectory (taking into account the renormalization), which also can be found in Ref. [3],

$$
\left.\omega^{Q}\left(-\vec{q}_{i}^{2}\right)\right|_{\text {renorm }}=\int d^{2+2 \epsilon} k F_{\omega}\left(\vec{k}, \vec{q}_{i}\right),
$$

where

$$
F_{\omega}\left(\vec{k}, \vec{q}_{i}\right)=\frac{\bar{q}_{\mu}^{4} \mu^{-2 \epsilon}}{\pi^{1+\epsilon} \epsilon \Gamma(1-\epsilon)} \frac{2 n_{f}}{3 N_{c}} \frac{\vec{q}_{i}^{2}}{\vec{k}^{2}\left(\vec{q}_{i}-\vec{k}\right)^{2}}\left[1+\frac{6 \Gamma^{2}(2+\epsilon)}{\Gamma(4+2 \epsilon)}\left(\left(\frac{\vec{q}_{i}^{2}}{\mu^{2}}\right)^{\epsilon}-\left(\frac{\vec{k}^{2}}{\mu^{2}}\right)^{\epsilon}-\left(\frac{\left(\vec{q}_{i}-\vec{k}\right)^{2}}{\mu^{2}}\right)^{\epsilon}\right)\right] \text {. }
$$

Now, introducing a small cut-off $\lambda$ and making it tending to zero after taking the limit $\epsilon \rightarrow 0$, we can write $F_{r}=F_{r}\left(\theta\left(\lambda^{2}-\vec{k}^{2}\right)+\theta\left(\vec{k}^{2}-\lambda^{2}\right)\right)$. Then in the second region we can take the limit $\epsilon=0$ in (75), whereas the contribution of the first region exactly cancel the pieces of the trajectories coming from the integration regions $\vec{k}^{2} \leq \lambda^{2}$ and $\left(\vec{q}_{i}-\vec{k}\right)^{2} \leq \lambda^{2}$ in (76). Outside these regions we can take the limit $\epsilon=0$ also in $F_{\omega}\left(\vec{k}, \vec{q}_{i}\right)$. Thus we come to the kernel at $D=4$, where

$$
\begin{gathered}
F_{r}\left(\vec{q}_{1}, \vec{q}_{1}^{\prime} ; \vec{q}\right)=\frac{\alpha_{s}^{2}(\mu)}{16 \pi^{3}} \frac{2 N_{c} n_{f}}{3}\left\{2\left(\ln \left(\frac{\vec{k}^{2}}{\mu^{2}}\right)-\frac{5}{3}\right)\left(\frac{\vec{q}_{2}^{\prime 2}}{\vec{q}_{2}^{2} \vec{k}^{2}}+\frac{\vec{q}_{1}^{\prime 2}}{\vec{q}_{1}^{2} \vec{k}^{2}}-\frac{\vec{q}^{2}}{\vec{q}_{1}^{2} \vec{q}_{2}^{2}}\right)\right. \\
\left.+\frac{\vec{q}^{2}}{\vec{q}_{1}^{2} \vec{q}_{2}^{2}} \ln \left(\frac{\vec{q}^{4} \vec{k}^{4}}{\vec{q}_{1}^{2} \vec{q}_{2}^{2} \vec{q}_{1}^{\prime 2} \vec{q}_{2}^{\prime 2}}\right)-\left(\frac{\vec{q}_{2}^{\prime 2}}{\vec{q}_{2}^{2} \vec{k}^{2}}-\frac{\vec{q}_{1}^{\prime 2}}{\vec{q}_{1}^{2} \vec{k}^{2}}\right) \ln \left(\frac{\vec{q}_{1}^{2} \vec{q}_{2}^{\prime 2}}{\vec{q}_{2}^{2} \vec{q}_{1}^{\prime 2}}\right)\right\},
\end{gathered}
$$




$$
F_{\omega}\left(\vec{k}, \vec{q}_{i}\right)=-\frac{\alpha_{s}^{2}(\mu)}{16 \pi^{3}} \frac{2 N_{c} n_{f}}{3} \frac{\vec{q}_{i}^{2}}{\vec{k}^{2}\left(\vec{q}_{i}-\vec{k}\right)^{2}}\left(\ln \left(\frac{\vec{k}^{2}\left(\vec{q}_{i}-\vec{k}\right)^{2}}{\mu^{2} \vec{q}_{i}^{2}}\right)-\frac{5}{3}\right) .
$$

Of course, at that the virtual and real parts contain infrared singularities. We have to remember that the singularities must be regularized by limitations on integration regions discussed above or in an equivalent way.

Note that in the result of the transformation (62), (63)

$$
\begin{aligned}
F_{r}\left(\vec{q}_{1}, \vec{q}_{1}^{\prime} ; \vec{q}\right) \rightarrow \frac{\alpha_{s}^{2}(\mu)}{16 \pi^{3}} \frac{4 N_{c} n_{f}}{3} & \left\{\frac{\vec{q}_{2}^{\prime 2}}{\vec{q}_{2}^{2} \vec{k}^{2}}\left(\ln \left(\frac{\vec{k}^{2} \vec{q}_{2}^{2}}{\mu^{2} \vec{q}_{2}^{\prime 2}}\right)-\frac{5}{3}\right)+\frac{\vec{q}_{1}^{\prime 2}}{\vec{q}_{1}^{2} \vec{k}^{2}}\left(\ln \left(\frac{\vec{k}^{2} \vec{q}_{1}^{2}}{\mu^{2} \vec{q}_{1}^{\prime 2}}\right)-\frac{5}{3}\right)\right. \\
& \left.-\frac{\vec{q}^{2}}{\vec{q}_{1}^{2} \vec{q}_{2}^{2}}\left(\ln \left(\frac{\vec{q}_{1}^{2} \vec{q}_{2}^{2}}{\vec{q}^{2} \mu^{2}}\right)-\frac{5}{3}\right)\right\},
\end{aligned}
$$

whereas the trajectory remains unchanged. Omitting the terms with $\delta\left(\vec{r}_{1}^{\prime}-\vec{r}_{2}^{\prime}\right)$ we can write the transformed kernel $\tilde{\mathcal{K}}^{Q}$ in the coordinate representation as

$$
\begin{gathered}
\left\langle\vec{r}_{1} \vec{r}_{2}\left|\tilde{\mathcal{K}}_{d}^{Q}\right| \vec{r}_{1}^{\prime} \vec{r}_{2}^{\prime}\right\rangle=\frac{\alpha_{s}^{2}(\mu) N_{c} n_{f}}{12 \pi^{3}}\left[\delta\left(\vec{r}_{1}-\vec{r}_{1}^{\prime}\right) \delta\left(\vec{r}_{2}-\vec{r}_{2}^{\prime}\right) \int d^{2} \rho g_{0}\left(\vec{r}_{1}, \vec{r}_{2} ; \rho\right)\right. \\
\left.+\delta\left(\vec{r}_{1}-\vec{r}_{1}^{\prime}\right) g\left(\vec{r}_{1}, \vec{r}_{2} ; \vec{r}_{2}^{\prime}\right)+\delta\left(\vec{r}_{2}-\vec{r}_{2}^{\prime}\right) g\left(\vec{r}_{2}, \vec{r}_{1} ; \vec{r}_{1}^{\prime}\right)\right]
\end{gathered}
$$

Let us define

$$
\begin{aligned}
& g\left(\vec{r}_{1}, \vec{r}_{2}\right)=\int \frac{d^{2} \rho}{(2 \pi)} \frac{d^{2} k}{(2 \pi)} \frac{d^{2} q}{(2 \pi)} \frac{d^{2} q^{\prime}}{(2 \pi)} {\left[\frac{1}{\vec{q}^{2}} \ln \frac{\vec{k}^{2}}{\vec{q}^{\prime 2}}+\frac{1}{\vec{k}^{2}} \ln \frac{\vec{q}^{2}}{\vec{q}^{\prime 2}}+\frac{\vec{k} \vec{q}}{\vec{k}^{2} \vec{q}^{2}}\left(\ln \left(\frac{\vec{k}^{2} \vec{q}^{2}}{\vec{q}^{\prime 2} \mu^{2}}\right)-\frac{5}{3}\right)\right] } \\
& \times e^{i \vec{k}\left(\vec{r}_{1}-\vec{\rho}\right)+i \vec{q}\left(\vec{r}_{2}-\vec{\rho}\right)-i \vec{q}^{\prime}\left(\vec{r}_{2}^{\prime}-\vec{\rho}\right)}
\end{aligned}
$$

Then the function $g\left(\vec{r}_{1}, \vec{r}_{2} ; \vec{r}_{2}^{\prime}\right)$ can be written as

$$
g\left(\vec{r}_{1}, \vec{r}_{2} ; \vec{r}_{2}^{\prime}\right)=g\left(\vec{r}_{1}, \vec{r}_{2}\right)-\frac{1}{2} g\left(\vec{r}_{2}, \vec{r}_{2}\right)-\frac{1}{2} g\left(\vec{r}_{1}, \vec{r}_{1}\right)
$$

Here the first term comes from the real part, the second from the trajectory, and the third is added "by hand", because its contribution is zero due to the gauge invariance. It is not a complicated task to calculate $g\left(\vec{r}_{1}, \vec{r}_{2}\right)$. The only integral which is not trivial is

$$
\int \frac{d^{2} k}{(2 \pi)} \frac{d^{2} q}{(2 \pi)} \frac{\vec{k} \vec{q}}{\vec{k}^{2} \vec{q}^{2}} \ln (\vec{k}+\vec{q})^{2} e^{i \vec{k} \vec{r}_{1}+i \vec{q}_{2}}=\frac{\vec{r}_{1} \vec{r}_{2}}{\vec{r}_{1}^{2} \vec{r}_{2}^{2}}\left(-2 \psi(1)-2 \ln 2+\ln \left(\frac{\vec{r}_{1}^{2} \vec{r}_{2}^{2}}{\left(\vec{r}_{1}-\vec{r}_{2}\right)^{2}}\right)\right) .
$$


As a result we have

$$
\begin{gathered}
g\left(\vec{r}_{1}, \vec{r}_{2}\right)=-2 \frac{\left(\vec{r}_{1}-\vec{r}_{2}^{\prime}\right)\left(\vec{r}_{2}-\vec{r}_{2}^{\prime}\right)}{\left(\vec{r}_{1}-\vec{r}_{2}^{\prime}\right)^{2}\left(\vec{r}_{2}-\vec{r}_{2}^{\prime}\right)^{2}} \ln \left(\frac{\vec{r}_{\mu}^{2}}{\left(\vec{r}_{1}-\vec{r}_{2}\right)^{2}}\right) \\
+\frac{1}{\left(\vec{r}_{1}-\vec{r}_{2}^{\prime}\right)^{2}} \ln \left(\frac{\left(\vec{r}_{2}-\vec{r}_{2}^{\prime}\right)^{2}}{\left(\vec{r}_{1}-\vec{r}_{2}\right)^{2}}\right)+\frac{1}{\left(\vec{r}_{2}-\vec{r}_{2}^{\prime}\right)^{2}} \ln \left(\frac{\left(\vec{r}_{1}-\vec{r}_{2}^{\prime}\right)^{2}}{\left(\vec{r}_{1}-\vec{r}_{2}\right)^{2}}\right)
\end{gathered}
$$

and $g\left(\vec{r}_{1}, \vec{r}_{2} ; \vec{r}_{2}^{\prime}\right)=f_{b}\left(\vec{r}_{1}, \vec{r}_{2} ; \vec{r}_{2}^{\prime}\right)$, with $f_{b}$ given in (72).

To find the function $g_{0}$ is an even simpler task. Using the representation

$$
\int d^{2} k \frac{2}{\vec{k}^{2}}\left(\ln \frac{\vec{k}^{2}}{\mu^{2}}-\frac{5}{3}\right)=-2 \int d^{2} \rho \frac{d^{2} k}{(2 \pi)} \frac{d^{2} q}{(2 \pi)} \frac{\vec{k} \vec{q}}{\vec{k}^{2} \vec{q}^{2}}\left(\frac{1}{2} \ln \frac{\vec{k}^{2} \vec{q}^{2}}{\mu^{4}}-\frac{5}{3}\right) e^{i \vec{k}\left(\vec{r}_{1}-\vec{\rho}\right)+i \vec{q}\left(\vec{r}_{2}-\vec{\rho}\right)}
$$

we have for the real part contribution $g_{0}\left(\vec{r}_{1}, \vec{r}_{2}\right)$ to $g_{0}\left(\vec{r}_{1}, \vec{r}_{2} ; \rho\right)$ :

$$
g_{0}\left(\vec{r}_{1}, \vec{r}_{2}\right)=\frac{\left(\vec{r}_{1}-\vec{\rho}\right)\left(\vec{r}_{2}-\vec{\rho}\right)}{\left(\vec{r}_{1}-\vec{\rho}\right)^{2}\left(\vec{r}_{2}-\vec{\rho}\right)^{2}} \ln \left(\frac{\vec{r}_{\mu}^{4}}{\left(\vec{r}_{1}-\vec{\rho}\right)^{2}\left(\vec{r}_{2}-\vec{\rho}\right)^{2}}\right) .
$$

It can be easily seen that the contribution of the virtual part is $-\left(g\left(\vec{r}_{1}, \vec{r}_{1}\right)+g\left(\vec{r}_{2}, \vec{r}_{2}\right)\right) / 2$, and taking into account that

$$
\begin{gathered}
\int \frac{d^{2} \rho}{\left(\vec{r}_{1}-\vec{\rho}\right)^{2}\left(\vec{r}_{2}-\vec{\rho}\right)^{2}}\left[\left(\vec{r}_{1}-\vec{r}_{2}\right)^{2} \ln \left(\frac{\left(\vec{r}_{1}-\vec{\rho}\right)^{2}\left(\vec{r}_{2}-\vec{\rho}\right)^{2}}{\left(\vec{r}_{1}-\vec{r}_{2}\right)^{4}}\right)\right. \\
\left.+\left(\left(\vec{r}_{1}-\vec{\rho}\right)^{2}-\left(\vec{r}_{2}-\vec{\rho}\right)^{2}\right) \ln \left(\frac{\left(\vec{r}_{1}-\vec{\rho}\right)^{2}}{\left(\vec{r}_{2}-\vec{\rho}\right)^{2}}\right)\right]=0,
\end{gathered}
$$

we can put $g_{0}\left(\vec{r}_{1}, \vec{r}_{2} ; \rho\right)=-f_{b}\left(\vec{r}_{1}, \vec{r}_{2} ; \rho\right)$, with $f_{b}$ given in (72). So, we reached the result of the previous Section for $D=4$ in a much shorter way.

\section{Conclusion}

The coordinate representation of the BFKL kernel is extremely interesting, because it gives the possibility to understand its conformal properties and the relation between the BFKL and the color dipole approaches. We performed the transformation to the coordinate representation of the quark contribution to the BFKL kernel in the next-to-leading order at large $N_{c}$ from the momentum representation where it was calculated before. Taking into account the freedom in the definition of the kernel, we found agreement with the result obtained recently in Ref. [14] by direct calculation of the quark contribution to the dipole kernel in the coordinate representation. 
The agreement is reached after some transformations of the original BFKL kernel which do not change the scattering amplitudes of color singlet objects. We have to add that in Ref. [14] also terms suppressed by $N_{c}^{2}$ are calculated. These terms have a very complicated form in the momentum representation [3]. On the contrary, in the coordinate representation they look quite simple. It would be very interesting to understand the reason for that.

As for the conformal properties of the NLO BFKL kernel, we have not found any representation in which the conformal invariance is violated in the NLA only by the renormalization.

When this article was in the stage of completion, a paper by Kovchegov and Weigart appeared [15]. Our results, obtained by a quite different approach, agree also with those of that paper.

\section{Acknowledgment}

V.S.F. thanks the Dipartimento di Fisica dell'Università della Calabria and the Istituto Nazionale di Fisica Nucleare, Gruppo Collegato di Cosenza, for the warm hospitality while part of this work was done and for the financial support.

\section{References}

[1] V.S. Fadin, E.A. Kuraev and L.N. Lipatov, Phys. Lett. B 60 (1975) 50; E.A. Kuraev, L.N. Lipatov and V.S. Fadin, Zh. Eksp. Teor. Fiz. 71 (1976) 840 [Sov. Phys. JETP 44 (1976) 443]; 72 (1977) 377 [45 (1977) 199]; Ya.Ya. Balitskii and L.N. Lipatov, Sov. J. Nucl. Phys. 28 (1978) 822.

[2] V.S. Fadin, L.N. Lipatov, Phys. Lett. B429 (1998) 127;

M. Ciafaloni and G. Camici, Phys. Lett. B430 (1998) 349.

[3] V.S. Fadin, R. Fiore and A. Papa, Phys. Rev. D60 (1999) 074025.

[4] V.S. Fadin and D.A. Gorbachev, Pis'ma v Zh. Eksp. Teor. Fiz. 71 (2000) 322 [JETP Letters 71 (2000) 222]; Phys. Atom. Nucl. 63 (2000) 2157 [Yad. Fiz. 63 (2000) 2253].

[5] V.S. Fadin and R. Fiore, Phys. Lett. B610 (2005) 61 [Erratum-ibid. B621 (2005) 61]; Phys. Rev. D72 (2005) 014018.

[6] L.N. Lipatov, Sov. Phys. JETP 63 (1986) 904 [Zh. Eksp. Teor. Fiz. 90 (1986) 1536]. 
[7] N.N. Nikolaev and B.G. Zakharov, Z. Phys. C64 (1994) 631;

N.N. Nikolaev, B.G. Zakharov and V.R. Zoller, JETP Lett. 59 (1994) 6;

A.H. Mueller, Nucl. Phys. B415 (1994) 373;

A.H. Mueller and B. Patel, Nucl. Phys. B425 (1994) 471.

[8] L.V. Gribov, E.M. Levin and M.G. Ryskin, Phys. Rep. 170 (1983) 1.

[9] I. Balitsky, Nucl. Phys. B463 (1996) 99;

Yu. Kovchegov, Phys. Rev. D60 (1999) 034008.

[10] L. McLerran and R. Venugopalan, Phys. Rev. D49 (1994) 2233; ibid. D49 (1994) 3352; ibid. D50 (1994) 2225;

E. Iancu, A. Leonidov and L. McLerran, Phys. Lett. B510 (2001) 133; Nucl.Phys. A692 (2001) 583;

E. Ferreiro, E. Iancu, A. Leonidov and L. McLerran, Nucl. Phys. A703 (2002) 489;

J. Jalilian-Marian, A. Kovner, A. Leonidov and H. Weigert, Nucl. Phys. B504 (1997) 415;

Phys. Rev. D59 (1999) 014014.

[11] J. Bartels, L.N. Lipatov and G.P. Vacca, Nucl. Phys. B706 (2005) 391;

J. Bartels, L.N. Lipatov, M. Salvadore and G.P. Vacca, Nucl. Phys. B726 (2005) 53.

[12] V.S. Fadin and R. Fiore, Phys. Lett. B440 (1998) 359.

[13] V.S. Fadin, R. Fiore and A. Papa, to be published.

[14] I. Balitsky, arXiv:hep-ph/0609105.

[15] Yu.V. Kovchegov and H. Weigert, arXiv:hep-ph/0612071. 